\title{
Paragangliomas múltiples asociados a mutación del gen SDHB. Caso clínico
}

\author{
RENÉ E. DÍAZ ${ }^{1,2}$, CARLOS UTRERAS ${ }^{2}$, RODRIGO ASCUÍ2, \\ FERNANDO HIDALGO ${ }^{2,3}$, JESÚS VÉLIZ ${ }^{1,2}$, NELSON WOHLLK ${ }^{1,2}$.
}

\section{Multiple paragangliomas associated to a $S D H B$ gene mutation. Report of one case}

Paragangliomas are tumors arising from sympathetic and parasympathetic tissues. The classic associated syndromes are neurofibromatosis type 1, multiple endocrine neoplasia type 2 and von Hippel-Lindau. Germline mutations of succinate dehydrogenase subunits genes, are associated with familial paraganglioma syndromes 1, 2, 3 and 4. We report a 29-year-old woman with a family background of pheochromocytoma and history of paroxysmal headache, nausea, sweating, palpitations, associated with severe hypertension. The patient had elevated plasma noradrenalin and urinary normetanephrines. Imaging studies revealed three retroperitoneal extra-adrenal masses. The clinical and laboratory study of classic syndromes associated with paraganglioma was negative. The patient was operated and the pathological study of the surgical specimen was consistent with paragangliomas. The genetic study showed a mutation in the SDHB succinate dehydrogenase gen, Exon 2 of CCTCA c.300_304 (p.P56delYfsX5).

(Rev Med Chile 2011; 139: 1475-1480).

Key words: Gene; Germ-line mutation; Paraganglioma; Pheochromocytoma; Succinate dehydrogenase.

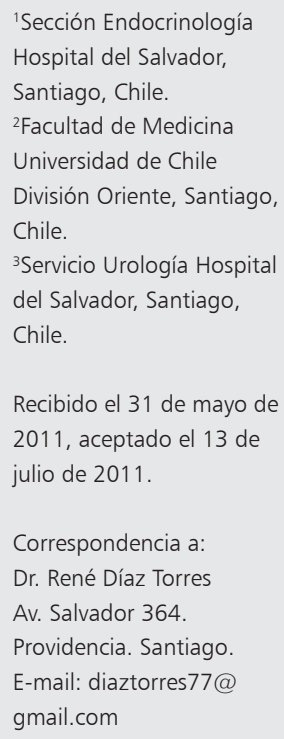

L os paragangliomas $(\mathrm{PG})$ son tumores de los paraganglios,que derivan de tejido simpático (secretores) cuando se ubican en localización adrenal o extraadrenal y de tejido parasimpático (no secretores) cuando se ubican en cabeza y cuello. Se denomina feocromocitoma (FEO) al tumor derivado de la médula adrenal que es el principal paraganglio simpático ${ }^{1}$. Las ubicaciones más frecuente de los PG son: el cuerpo de Zuckerkandl, en relación a la arteria mesentérica superior, los plexos simpáticos de la vejiga, riñón, corazón, mediastino, cabeza y cuello; estos últimos generalmente parasimpáticos y por lo tanto no secretores de catecolaminas.

La incidencia de FEO/PG es $<$ 1:300.000/año y clínicamente se presentan con hipertensión asociada a síntomas paroxísticos (taquicardia, cefalea, palpitaciones y sudoración) espontáneos o provocados por aumento de la presión intraabdominal, o mínimamente sintomáticos, siendo diagnosti- cados al estudiar un incidentaloma suprarrenal ${ }^{1}$. Existe asociación importante de FEO/PG con síndromes genéticos hereditarios y se considera actualmente que $30 \%$ se encuentran en el contexto de alguna mutación genética ${ }^{1}$. Avances importantes al respecto se han realizado en la última década lo que ha facilitado la comprensión, diagnóstico y seguimiento de esta patología.

Presentamos el caso de una paciente portadora de paragangliomas múltiples en la cual se realizó estudio genético, enfatizando la utilidad de realizarlo en pacientes seleccionados y su relevancia en el tratamiento y seguimiento de estos pacientes y sus familias.

\section{Caso clínico}

Paciente de sexo femenino de 29 años, procedente de Calama. Dentro de sus antecedentes destaca trombosis retinal de ojo izquierdo 2 meses 
previo al cuadro actual y madre operada de feocromocitoma a los 26 años de edad. Historia de 10 años de crisis paroxísticas de cefalea, naúseas, sudoración, palpitaciones, asociadas a hipertensión arterial severa.

En tratamiento hace 6 años con beta bloqueadores e inhibidores de enzima convertidora de angiotensina. Por aumento en la frecuencia de las crisis fue hospitalizada destacando al examen físico: presión arterial 158/111 $\mathrm{mmHg}$, pulso 93 por minuto, examen cardiopulmonar normal, abdomen blando, depresible, sin masas palpables.

Exámenes de ingreso; creatinina $0,75 \mathrm{mg} / \mathrm{dL}$, calcemia $10,3 \mathrm{mg} / \mathrm{dL}$, fosfemia $3,5 \mathrm{mg} / \mathrm{dL}$, TSH $0,45 \mathrm{uUI} / \mathrm{mL}(0,4-4,0)$, T4 libre $0,91 \mathrm{ng} / \mathrm{dL}(0,8$ $1,8)$, aldosterona plasmática $22,7 \mathrm{ng} / \mathrm{dL}(1,0-16)$, actividad renina plasmática $20 \mathrm{ng} / \mathrm{mL} / \mathrm{h}(1,6 \pm$ $0,83)$, noradrenalina plasmática $7192 \mathrm{pg} / \mathrm{mL}$ (41$773)$, adrenalina plasmática $<20 \mathrm{pg} / \mathrm{mL}(20-82)$, normetanefrinas urinarias $4816 \mathrm{ug} / \mathrm{g}$ creatinina (46-256), metanefrinas urinarias $122 \mathrm{ug} / \mathrm{g}$ creatinina (25-155), calcitonina plasmática ultrasensible $<0,2 \mathrm{pg} / \mathrm{mL}(<7,5)$, antígeno carcinoembrionario $1,14 \mathrm{ng} / \mathrm{mL}(<4,2)$. Las mediciones de estas hormonas fueron realizadas bajo tratamiento con amlodipino, el cual tiene mínima influencia sobre el eje renina-angiotensina-aldosterona y no afecta la medición de las metanefrinas urinarias ${ }^{2,3}$.

Ecografía tiroidea evidenció nódulos tiroideos bilaterales de aspecto coloideo, el mayor en lóbulo derecho de $10 \mathrm{~mm}$. Tomografía computada (TAC) de abdomen y angioTAC renal mostraron 3 masas retroperitoneales: una pararrenal izquierda de 8 $\mathrm{cm}$, otra en hilio renal izquierdo de $3 \mathrm{~cm}$ y la tercera paraaórtica de $4 \mathrm{~cm}$, además de cierto grado de compresión de vena cava inferior y presencia de dos arterias renales izquierda (Figuras 1 y 2). Fondo de ojo y resonancia magnética (RM) de cerebro y cuello descartaron presencia de hemangiomas retinales y hemangioblastomas cerebrales. En base a la ausencia de elementos clínicos sugerentes de neurofibromatosis tipo 1 (NF1), neoplasia endocrina múltiple tipo 2 (NEM 2) y de enfermedad de von Hippel-Lindau (VHL), la presencia de masas extraadrenales y considerando el antecedente materno de feocromocitoma, se planteó el diagnóstico de síndrome de paraganglioma hereditario.

El estudio genético de la paciente, realizado en el laboratorio del Prof. Hartmut P.H. Neumann (Universidad de Freiburg, Alemania) mostró una mutación del gen succinato deshidrogenasa SDHB

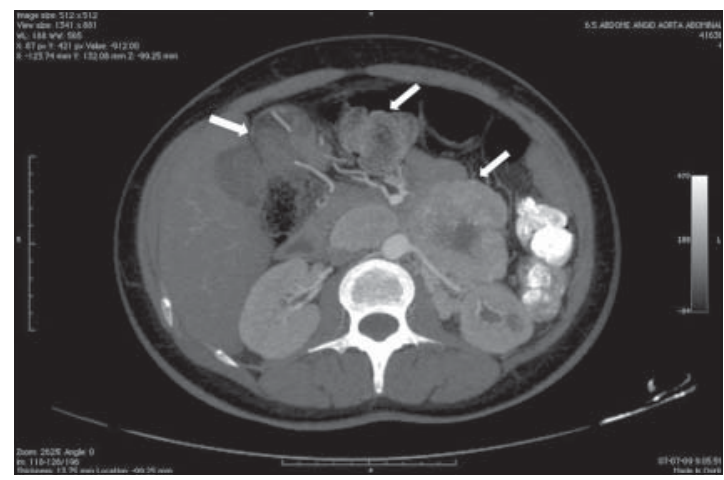

Figura 1. Tomografía computada (TAC) de abdomen. Los paragangliomas se identifican en la figura con flechas blancas.

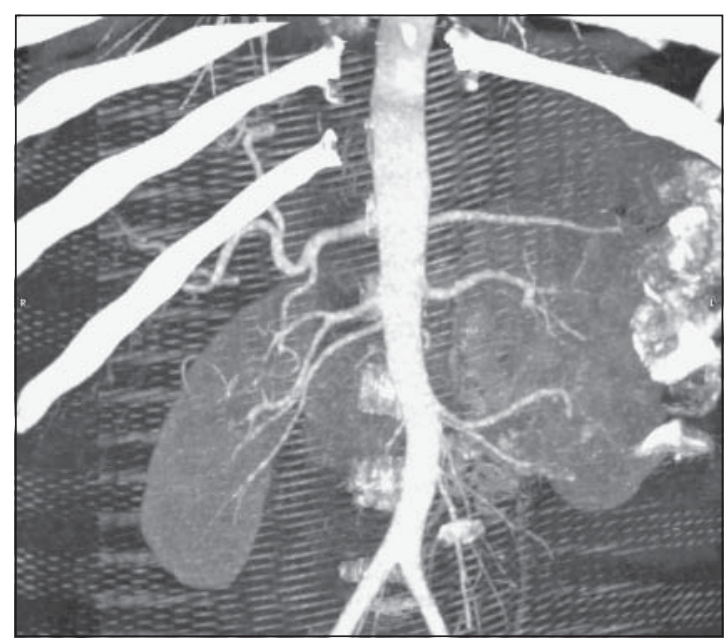

Figura 2. AngioTAC renal.

Exon 2 c.300_304 del CCTCA (p.P56delYfsX5), siendo negativo en su hija.

Se realizó cirugía, resecándose 3 masas retroperitoneales que se correspondían en ubicación y tamaño con la TAC abdominal. El estudio histológico informó paragangliomas. Las normetanefrinas urinarias se normalizaron y las resonancias magnéticas de cabeza, cuello, abdomen y pelvis, además de TAC de Tórax fueron normales.

\section{Discusión}

Tradicionalmente se ha aplicado al FEO/PG la "regla de los 10" (10\% bilaterales, $10 \%$ malignos, $10 \%$ extraabdominales, $10 \%$ extraadrenales, $10 \%$ normotensos y $10 \%$ de origen genético), siendo el 
componente genético atribuible a los síndromes de NEM 2, VHL y NF $1^{4}$. Sin embargo, la descripción de mutaciones en los genes de SDH ha configurado los síndromes de paragangliomas familiares tipo 1, 3 y 4 (PGL 1, PGL3 y PGL 4) que corresponden a mutaciones germinales de las subunidades $\mathrm{D}, \mathrm{C}$ y $\mathrm{B}$ de SDH (SDHD, SDHC y SDHB) respectivamente, lo cual ha conducido a una revisión de la "regla de los 10", llegando a la conclusión de que 20-25\% de los pacientes con $\mathrm{FEO} / \mathrm{PG}$ aparentemente esporádicos, son de origen genético ${ }^{5-7}$.

En una serie del grupo de trabajo del Dr. HP Neumann de 1.149 pacientes con FEO/PG se encontró una mutación de línea germinal en 30,1\% de los casos, donde los síndromes hereditarios clásicamente asociados con FEO/PG dieron cuenta de $70 \%$ (34,7\% VHL, $23,1 \%$ NEM 2 y $12,4 \%$ NF1), mientras que el $30 \%$ restante correspondieron a mutaciones del gen SDH, principalmente SDHB $(21,1 \%)$ y SDHD $(8,1 \%)$, con sólo dos casos de mutación de SDHC $(0,5 \%)^{8}$.

La SDH es una enzima mitocondrial que participa tanto en el ciclo del ácido tricarboxílico como en la cadena de transporte de electrones (complejo mitocondrial II), contribuyendo a la formación de ATP por fosforilación oxidativa; por lo tanto juega un rol crucial en la generación de energía. En este complejo enzimático, el núcleo y principal dominio catalítico está formado por SDHA y SDHB, teniendo las SDHC y SDHD un rol en la transferencia de electrones y en el anclaje a la membrana?.

Como se ha señalado anteriormente, mutaciones asociadas a FEO/PG se han encontrado en las subunidades SDHB, SDHC y SDHD, con características fenotípicas marcadamente distintas y en los últimos años han sido descritas nuevas mutaciones, entre ellas la primera mutación del gen de la subunidad A de SDH (SDHA) en una paciente con PG secretor abdominal, además de la descripción de la mutación de SDHAF2 que es un cofactor para la flavinización de SDHA, paso esencial para la correcta función del complejo $\mathrm{SDH}$, que también ha sido asociado a la presencia de $\mathrm{PG}^{10,11}$.

Recientemente se ha publicado una mutación de un gen supresor de tumores que codifica para un proteína transmembrana llamada TMEM127, la que a diferencia de $\mathrm{SDH}$, no posee dominios funcionales y se ha asociado exclusivamente a $\mathrm{FEO}^{12}$.
Cabe señalar que todos los genes de SDH y de VHL son genes supresores de tumores y que la mutación germinal induce la pérdida del otro alelo en el tumor (pérdida de la heterocigosidad), hallazgos consistentes con el concepto de Knudson (two hits) de inactivación bialélica de un gen supresor de tumor. La herencia es autosómica dominante en el caso de mutaciones de SDHB y SDHC, mientras que la susceptibilidad es de transmisión paterna en el caso de SDHD y SDHAF $2^{9}$.

Es relevante tener clara la presentación clínica de cada uno de los síndromes genéticos asociados a FEO/PG con la finalidad de contar con predictores clínicos que permitan identificar qué pacientes deben ser estudiados y qué mutación debe ser investigada, como se resume en la Tabla $1^{10,11,13-23}$.

La sintomatología depende fundamentalmente del tipo de catecolamina secretada (fenotipo) y de la susceptibilidad individual. Los PG parasimpáticos presentan sintomatología derivada del efecto de masa o infiltración del tumor y son hormonalmente silentes ya que no producen catecolaminas. Esto implica que el diagnóstico sea más tardío. Los FEO/PG en VHL son menos sintomáticos que los asociados a NEM 2 ya que poseen menor expresión de tirosina hidroxilasa y n-metiltransferasa lo que se traduce en menor secreción de metanefrinas y mayor secreción de normetanefrinas ${ }^{1}$. Es así como se reconoce el fenotipo adrenérgico, caracterizado por sintomatología episódica (cefalea, palpitaciones y diaforesis) y episodios de hipertensión arterial paroxística como es el caso de NEM 2 y el fenotipo noradrenérgico que se caracteriza por hipertensión arterial sostenida que es lo que sucede en VHL.

Se han identificado los siguientes predictores de FEO/PG asociados a síndromes genéticos: edad de aparición $\leq 45$ años, tumores múltiples, ubicación extraadrenal, presencia de paragangliomas de cabeza y cuello, tumores malignos e historia familiar ${ }^{22}$. Esto ha conducido al desarrollo de algoritmos para el estudio de estos pacientes (Figura 3$)^{8}$.

Recientes investigaciones han aportado importante información para el estudio genético de los PG. Se ha visto que la pérdida de cualquier componente del complejo mitocondrial II (SDH) produce inestabilidad de dicho complejo lo que se traduce en la pérdida de expresión de SDHB en la inmunohistoquímica. Este hallazgo obligaría al estudio de los genes SDHB, SDHC y SDHD para 


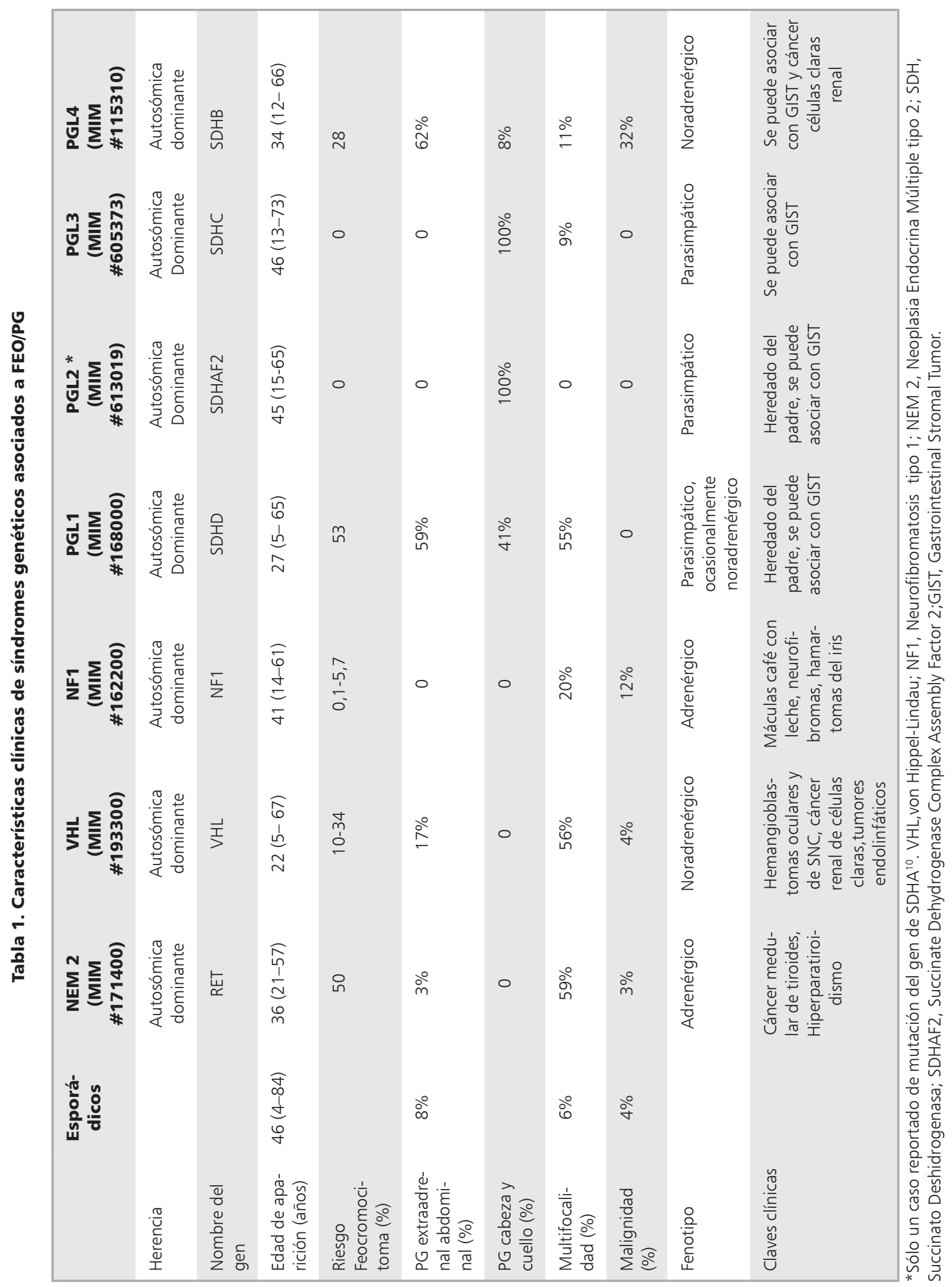




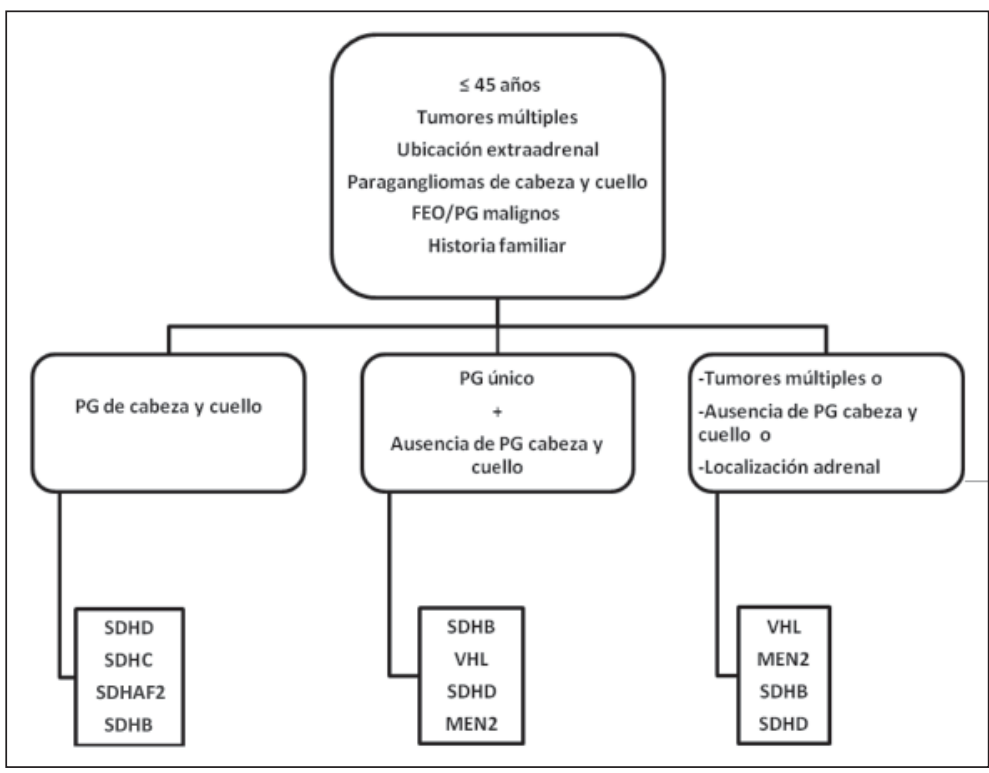

Figura 3. Algoritmo de estudio feocromocitomas de origen genético. Adaptado de referencia 8. confirmar o descartar el origen hereditario ${ }^{24-26}$.

Nuestra paciente debutó a los 29 años con HTA, siendo importante el antecedente familiar de FEO y presencia de PG funcionales múltiples extraadrenales, en ausencia de signos clínicos de NEM 2 y VHL, por lo que se realizó estudio de mutaciones del gen de SDH, resultando positiva para SDHB. Con esta información, se hizo búsqueda de PG de cabeza, cuello y tórax la cual resultó negativa, evolucionando con normalización de normetanefrinas urinarias y sin evidencias de recidiva. Hay que considerar sin embargo, que la mutación de SDHB le confiere un mayor potencial maligno por lo que deberá realizarse un estudio anual con metanefrinas urinarias y RM de cuello, tórax, abdomen y pelvis ${ }^{19}$.

\section{Referencias}

1. Opocher G, Schiavi F. Genetics of pheochromocytomas and paragangliomas. Best Pract Res Clin Endocrinol Metab 2010; 24: 943-56.

2. Rossi GP. A comprehensive review of the clinical aspects of primary aldosteronism. Nat Rev Endocrinol. Published online 24 May 2011.

3. Eisenhofer G, Goldstein DS, Walther MM, Friberg P, Lenders JW, Keiser HR, et al. Biochemical diagnosis of pheochromocytoma: how to distinguish true-from falsepositive test results. J Clin Endocrinol Metab 2003; 88:
2656-66.

4. Bravo EL, Gifford RW Jr. Current concepts. Pheochromocytoma: diagnosis, localization and management. $\mathrm{N}$ Engl J Med 1984; 311: 1298-303.

5. Baysal BE, Ferrell RE, Willett-Brozick JE, Lawrence EC, Myssiorek D, Bosch A, et al. Mutations in SDHD, a mitochondrial complex II gene, in hereditary paraganglioma. Science 2000; 287: 848-51.

6. Neumann HP, Bausch B, Mcwhinney SR, Bender BU, Gimm O, Franke G, et al. Germ-line mutations in non syndromic pheochromocytoma. N Engl J Med 2002; 346: 1459-66.

7. Dluhy RG. Pheochromocytoma-Death of an Axiom. N Engl J Med 2002; 346: 1486-8.

8. Erlic Z, Rybicki L, Peczkowska M, Golcher H, Kann PH, Brauckhoff M, et al. Clinical Predictors and Algorithm for the Genetic Diagnosis of Pheochromocytoma Patients. Clin Cancer Res 2009; 15: 6378-85.

9. Hensen EF, Bayley JP. Recent advances in the genetics of SDH-related paraganglioma and pheochromocytoma. Fam Cancer 2011; 10: 355-63.

10. Burnichon N, Brière JJ, Libé R, Vescovo L, Rivière J, Tissier $\mathrm{F}$, et al. SDHA is a tumor suppressor gene causing paraganglioma. Hum Mol Genet 2010; 19: 3011-20.

11. Hao HX, Khalimonchuk O, Schraders M, Dephoure N, Bayley JP, Kunst H, et al. SDH5, a gene required for flavination of succinate dehydrogenase, is mutated in paraganglioma. Science 2009; 325: 1139-42.

12. Qin Y, Yao L, King EE, Buddavarapu K, Lenci RE, Cho- 
cron ES, et al. Germline mutations in TMEM127 confer susceptibility to pheochromocytoma. Nat Genet 2010; 42: 229-33.

13. Walther MM, Herring J, Enquist E, Keiser HR, Linehan WM. Von Recklinghausen's disease and pheochromocytomas. J Urol 1999; 162: 1582-86.

14. Hes FJ, Höppener JW, Lips CJ. Clinical review 155: Pheochromocytoma in Von Hippel-Lindau disease. J Clin Endocrinol Metab 2003; 88: 969-74.

15. O'Riordain DS, O'Brien T, Crotty TB, Gharib H, Grant CS, van Heerden JA. Multiple endocrine neoplasia type 2B: more than an endocrine disorder. Surgery 1995; 118 : 936-42.

16. Leboulleux S, Travagli JP, Caillou B, Laplanche A, Bidart JM, Schlumberger M, et al. Medullary thyroid carcinoma as part of a multiple endocrine neoplasia type $2 \mathrm{~B}$ syndrome: influence of the stage on the clinical course. Cancer 2002; 94: 44-50.

17. Neumann HP, Pawlu C, Peczkowska M, Bausch B, McWhinney SR, Muresan M, et al. Distinct clinical features of paraganglioma syndromes associated with SDHB and SDHD gene mutations. JAMA 2004; 292: 943-51.

18. Niemann S, Steinberger D, Müller U. PGL3, a third, not maternally imprinted locus in autosomal dominant paraganglioma. Neurogenetics 1999; 2: 167-70.

19. Timmers HJ, Gimenez-Roqueplo AP, Mannelli M, Pacak K. Clinical aspects of SDHx-related pheochromocytoma and paraganglioma. Endocrine-Related Cancer 2009; 16: 391-400.
20. Schiavi F, Milne RL, Anda E, Blay P, Castellano M, Opocher $\mathrm{G}$, et al Are we overestimating the penetrance of mutations in SDHB? Hum Mutat 2010; 31: 761-62.

21. Neumann HP, Eng C. The Approach to the Patient with Paraganglioma J Clin Endocrinol Metab 2009; 94: 2677 83

22. Mannelli M, Castellano $M$, Schiavi F, Filetti $S$, Giacchè M, Mori L, et al. Clinically Guided Genetic Screening in a Large Cohort of Italian Patients with Pheochromocytomas and/or Functional or Nonfunctional Paragangliomas. J Clin Endocrinol Metab 2009; 94: 1541-47.

23. Jiménez C, Cote G, Arnold A, Gagel RF. Review: Should patients with apparently sporadic pheochromocytomas or paragangliomas be screened for hereditary syndromes? J Clin Endocrinol Metab 2006; 91: 2851-58.

24. van Nederveen FH, Gaal J, Favier J, Korpershoek E, Oldenburg RA, de Bruyn EM, et al. An immunohistochemical procedure to detect patients with paraganglioma and phaeochromocytoma with germline SDHB, SDHC, or SDHD gene mutations: a retrospective and prospective analysis. Lancet Oncol 2009; 10: 764-71.

25. Gill AJ, Benn DE, Chou A, Clarkson A, Muljono A, Meyer-Rochow GY, et al. Immunohistochemistry for SDHB triages genetic testing of SDHB, SDHC, and SDHD in paraganglioma-pheochromocytoma syndromes. Hum Pathol 2010; 41:805-14.

26. Gill AJ, Pachter NS, Clarkson A, Tucker KM, Winship IM, Benn DE, et al. Renal tumors and hereditary pheochromocytoma-paraganglioma syndrome type 4 . N Engl J Med 2011; 364: 885-6. 